\title{
Borohydride Oxidation at a Gold Electrode
}

\author{
Michael V. Mirkin, Hongjum Yang," and Allen J. Bard** \\ Department of Chemistry and Biochemistry, The University of Texas at Austin, Austin, Texas 78712
}

\section{ABSTRACT}

The multistage process of borohydride oxidation in an $8 e^{-}$reaction to borate at a Au electrode has been studied by means of fast-scan cyclic voltammetry (CV) and scanning electrochemical microscopy (SECM). The total irreversibility of this process observed previously is shown to be a result of the presence of very unstable intermediates. CV measurements showed that at least two stages of the process are quasi-reversible, and the presence of a coupled homogeneous chemical reaction was proved by SECM. The rate constant for this reaction as well as the electrochemical kinetic parameters for the first stage of oxidation are evaluated using digital simulation. The adsorption of the electroactive species associated with the first two-electron stage of the oxidation becomes apparent at scan rates higher than $200 \mathrm{~V} / \mathrm{s}$. A very small fractional surface coverage (estimated to be less than 0.001) is shown to produce CV waves characteristic of adsorption- rather than diffusion-controlled processes. The second chemical stage of this process is much faster than the first. The oxidation of borohydride at a gold anode is shown to have a different mechanism than that proposed earlier for a platinum electrode.

We describe here a study of the electrochemical oxidation of borohydride (or tetrahydroborate), $\mathrm{BH}_{4}^{-}$, at a gold electrode and the use of ultramicroelectrodes and fast techniques to study this complex process. Electrochemical oxidation of sodium borohydride and its derivatives in aqueous solutions was a subject of several detailed studies as described in a previous review ${ }^{1}$. In earlier studies ${ }^{2-8}$, carried out in the 1950s and 1960s, fast electrochemical techniques were not available to study rapid chemical stages and detect unstable intermediates. Interest in this process arises from its possible application as an anodic "fuel" in a battery, its role in electroless deposition (e.g., of $\mathrm{Au}$ ), and the challenge of elucidating a complicated (irreversible $8 e^{-}$) electrode reaction

$$
\mathrm{BH}_{4}^{-}+8 \mathrm{OH}^{-} \rightarrow \mathrm{BO}_{2}^{-}+6 \mathrm{H}_{2} \mathrm{O}+8 e^{-}
$$

Study of aqueous solutions of $\mathrm{BH}_{4}^{-}$is complicated by its rapid reaction with water (termed hydrolysis) at $\mathrm{pHs}$ below about 12

$$
\begin{aligned}
& \mathrm{BH}_{4}^{-}+\mathrm{H}_{2} \mathrm{O} \stackrel{k_{1}}{\rightarrow} \mathrm{BH}_{3} \mathrm{OH}^{-}+\mathrm{H}_{2} \\
& \mathrm{BH}_{3} \mathrm{OH}^{-}+\mathrm{H}_{2} \mathrm{O} \stackrel{k_{2}}{\rightarrow} \mathrm{BO}_{2}^{-}+3 \mathrm{H}_{2}
\end{aligned}
$$

The first reaction results in the appearance of $\mathrm{BH}_{3} \mathrm{OH}^{-}$, which can be oxidized at more negative potentials than borohydride. The product of the second reaction, borate, is not electroactive. A result of hydrolysis is the loss of $\mathrm{BH}_{4}^{-}$ and the appearance of the intermediate $\mathrm{BH}_{3} \mathrm{OH}^{-}$in solutions whose $\mathrm{pH}$ is not sufficiently high. The rates of these electron-transfer reactions strongly depend on the nature of the electrode material, and in particular its ability to catalyze hydrogen evolution. This becomes clear when comparing cyclic voltammetry (CV) results obtained at gold (noncatalytic) and platinum (catalytic) electrodes. The oxidation at $\mathrm{Au}$ occurs in a single well-shaped peak, but at $\mathrm{Pt}$ several overlapping peaks appear ${ }^{9}$. In spite of these differences, in most studies (excluding Ref. 8) $\mathrm{BH}_{3} \mathrm{OH}^{-}$ion was proposed as an intermediate during oxidation at $\mathrm{Pt}^{3}, \mathrm{Pd}^{4}, \mathrm{Au}^{7}$, or $\mathrm{Hg}^{5}$ anodes. This species can appear as a result of the hydrolysis ${ }^{57}$ or as a product of the first stage of the electro-oxidation process ${ }^{3,4}$. This intermediate was proposed to be bound to platinum ${ }^{3}$, as well as to the gold surface ${ }^{7}$.

Our previous studies ${ }^{9,10}$ showed that the choice of the noncatalytic gold electrode and a concentration of $\mathrm{OH}^{-}$ sufficiently high to eliminate hydrolysis provides the most reliable way for studying kinetics of the rather complicated oxidation of borohydride. To our knowledge the only previous study of this process with a $\mathrm{Au}$ anode was reported by Okinaka? ${ }^{7}$. The conditions of that experiment ( $T=75^{\circ} \mathrm{C}$ and $0.2 \mathrm{M} \mathrm{KOH}$ as a supporting electrolyte) allowed a significant rate of borohydride hydrolysis, and therefore a direct comparison to our results is not possible. The fast CV studies at a $\mathrm{Au}$ microelectrode reported here

\footnotetext{
* Electrochemical Society Student Member.

** Electrochemical Society Life Member.
}

allowed the direct observation, for the first time, of an intermediate in the anodic process. We found, however, that fast CV studies are limited by the effects of very small amounts of adsorption of reactant or intermediate, as well as a high sensitivity to heterogeneous kinetics. Generation/collection (G/C) experiments with the scanning electrochemical microscope (SECM) are also described.

\section{Experimental}

Reagents.- $\mathrm{NaBH}_{4}$ (Aldrich Chemical Company, Milwaukee, WI) and $\mathrm{NaOH}$ (Baker Analyzed, J. T. Baker Inc., Phillipsburg, NJ) were used without further purification. All solutions were prepared with deionized water (Milli-Q, Millipore Corp.)

Electrodes.-Two Au microdisks (12.5 and $50 \mu \mathrm{m}$ diam) encased in glass were used as working electrodes in CV experiments; the counterelectrode was a $5 \mathrm{~mm}$ diam Au disk. Before each CV experiment, the microdisk was polished on nylon cloth with $0.25 \mu \mathrm{m}$ diamond paste (Buehler Ltd, Lake Bluff, IL). Potentials were always measured vs. a $\mathrm{Hg} / \mathrm{Hg}_{2} \mathrm{SO}_{4} / \mathrm{sat} \mathrm{K}_{2} \mathrm{SO}_{4}$ reference electrode (SMSE).

The SECM tip was prepared from $10 \mu \mathrm{m}$ diam gold wire as described previously ${ }^{11}$. The substrate electrode (a $100 \mu \mathrm{m}$ diam gold disk sealed in a glass tube) was placed in the Teflon cell through the hole drilled in its bottom. It was critical for this experiment that the cell and electrodes were composed only of Teflon and glass without any kind of epoxy cement or other sealant, since preliminary experiments showed that a tiny amount of epoxy cement dissolves in a strongly alkaline solution and then adsorbs on and blocks the tip surface. Impurity adsorption on the much larger substrate surface of larger Au electrodes is less important. The use of a microelectrode substrate permitted biasing with a low current potentiostat with quasisteady-state conditions at the substrate during the generation/collection experiments. Both the tip and substrate electrodes were polished with $0.05 \mu \mathrm{m}$ alumina on felt (Buehler Ltd, Lake Bluff, IL). The auxiliary electrode in SECM was a $0.5 \mathrm{~mm}$ diam $\mathrm{Au}$ wire.

CV measurements.-The potential of the working electrode was controlled by a Princeton Applied Research (PAR, Princeton, NJ) Model 175 programmer. The slowscan voltammograms (scan rate, $v \leq 1 \mathrm{~V} / \mathrm{s}$ ) were obtained with a PAR Model 173 potentiostat. The fast-scan voltammograms were taken with a home-built potentiostat with a rise time of about $100 \mathrm{~ns}$ and recorded with a Norland 3001A/DMX digital processing oscilloscope (Norland Corporation, Fort Atkinson, WI). CV at $v=3 \times 10^{4} \mathrm{~V} / \mathrm{s}$ was recorded with a Tektronix 2440 digital oscilloscope (Tektronix, Inc., Beaverton, OR). A more detailed description of the apparatus is available ${ }^{12}$.

SECM apparatus.-A four-electrode potentiostat EL-400 (Ensman Instruments, Bloomington, IN) was used for independent control of the tip and substrate potentials. 


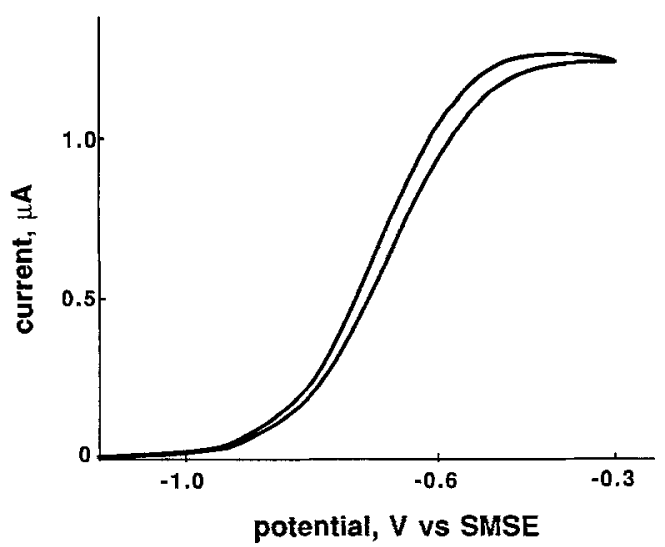

Fig. 1. Steady-state $\mathrm{CV}$ recorded at a $50 \mu \mathrm{m}$ diam gold microdisk for oxidation of $10 \mathrm{mM}$ sodium borohydride in $1 \mathrm{M} \mathrm{NaOH} ; \mathrm{v}=$ $10 \mathrm{mV} / \mathrm{s}$.

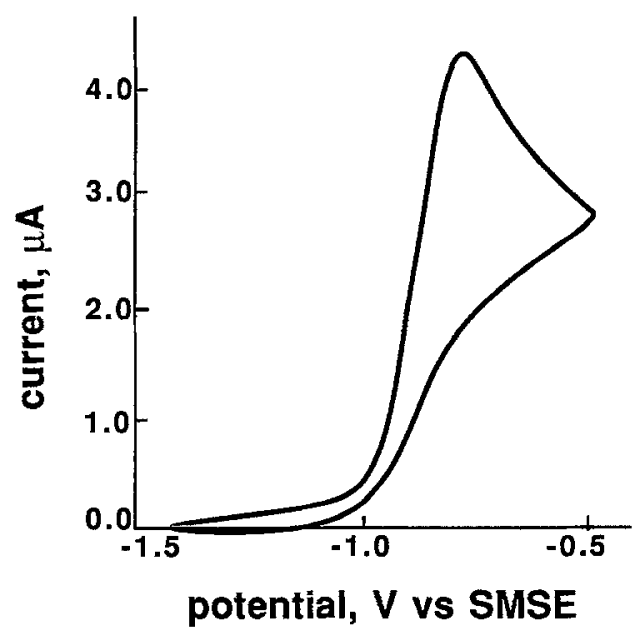

Fig. 2. As in Fig. $1 ; v=10 \mathrm{~V} / \mathrm{s}$.

Other details of the SECM apparatus have been described previously ${ }^{11,13,16}$.
SECM-procedure.-Unlike the previous work where SECM was used for kinetic measurements ${ }^{17,18}$, we could not work in a feedback mode ${ }^{19}$, because only very small or no positive feedback currents could be obtained with the highly unstable intermediate produced in this experiment. This small feedback current is difficult to measure on top of the much larger diffusion-controlled tip current flowing during a feedback SECM experiment. Thus the measurements were done in a $\mathrm{G} / \mathrm{C}$ mode ${ }^{13-15}$. The tip electrode biased at $-1.3 \mathrm{~V}$ (the potential at which no oxidation of $\mathrm{BH}_{4}^{-}$occurs, see Fig. 1) very slowly approached the substrate surface until a large current increase indicated electric contact between two working electrodes. The tip was then moved away from the substrate until a sharp decrease of the current was noted. This procedure allowed the minimum tip/substrate spacing achievable in this experiment. At this point the dependence of the tip current on substrate potential was recorded. Tip current/distance curves obtained with the same electrodes but in the presence of a reversible mediator $\left[\mathrm{K}_{3} \mathrm{Fe}(\mathrm{CN})_{6}\right]$ in the solution showed that the minimum tip/substrate distance in the G/C experiment was approximately $1 \mu \mathrm{m}$.

\section{Results and Discussion}

Cyclic voltammetry.-In a previous study ${ }^{9}$, we showed that slow scan $(100 \mathrm{mV} / \mathrm{s})$ voltammograms of borohydride oxidation at a gold electrode possess the shape typical of a simple irreversible electrode reaction. Analogously, with a microdisk electrode a well-defined steady-state $\mathrm{CV}$ is found (Fig. 1). In a concentrated alkaline solution $(1 \mathrm{M} \mathrm{NaOH})$, the rate of the borohydride hydrolysis is negligible $e^{5}$ and one can expect the number of electrons involved in this process $(n)$ to be close to 8 . In fact, using the borohydride diffusion coefficient value $D=1.6 \times$ $10^{-5} \mathrm{~cm}^{2} / \mathrm{s}^{10}$, we found $n \approx 7.9$ from the limiting current given by Eq. 4

$$
i_{\mathrm{T}}=4 n F a c^{*} D
$$

At higher $v$, a nonsteady-state CV (Fig. 2), which consists of a single anodic peak quite similar to that observed at a large electrode ${ }^{9}$, is obtained. A further increase in $v$ led to the noticeable change of the CV shape (Fig. 3a); a new anodic peak appeared at more negative potentials than the main wave, with a corresponding small cathodic peak on reversal. At $v=100 \mathrm{~V} / \mathrm{s}$ (Fig. $3 \mathrm{~b}$ ), the new anodic peak became better defined, while the cathodic peak increased.
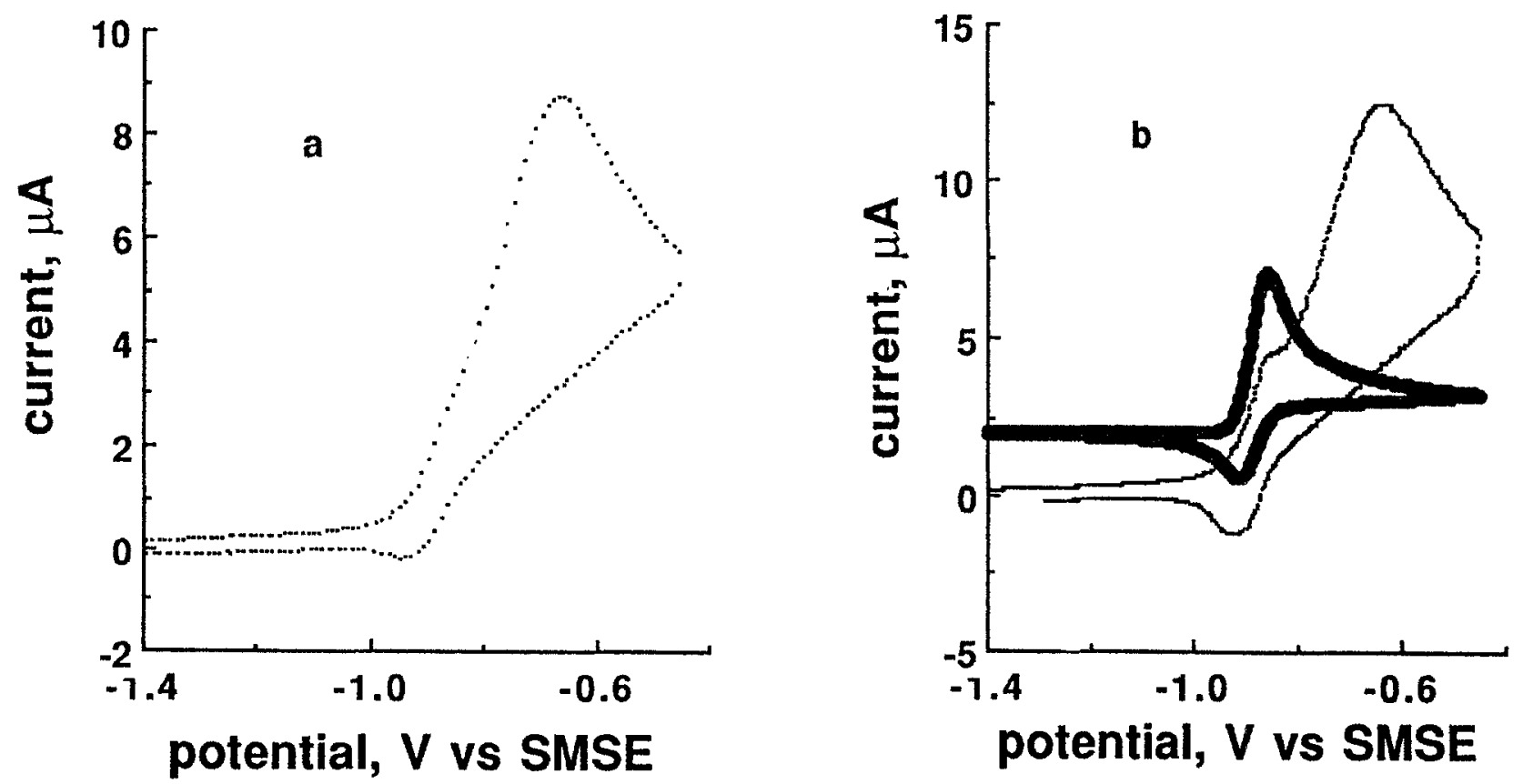

Fig. 3. As in Fig. $I ; v=50 \mathrm{~V} / \mathrm{s}$ (a) and $v=100 \mathrm{~V} / \mathrm{s}$ (b). The darker curve in (b) is a CV computed for a $E_{q} C_{i}$ mechanism (the first $2 \mathrm{e}^{-}$stage of the oxidation process) with the following values of parameters: $n=2, D=1.6 \times 10^{-5} \mathrm{~cm}^{2} / \mathrm{s}, k=0.3 \mathrm{~cm} / \mathrm{s} ; \alpha=0.5$, and $k=250 \mathrm{~s}^{-1}$ (offset on current axis by $2 \mu \mathrm{A}$ ). 

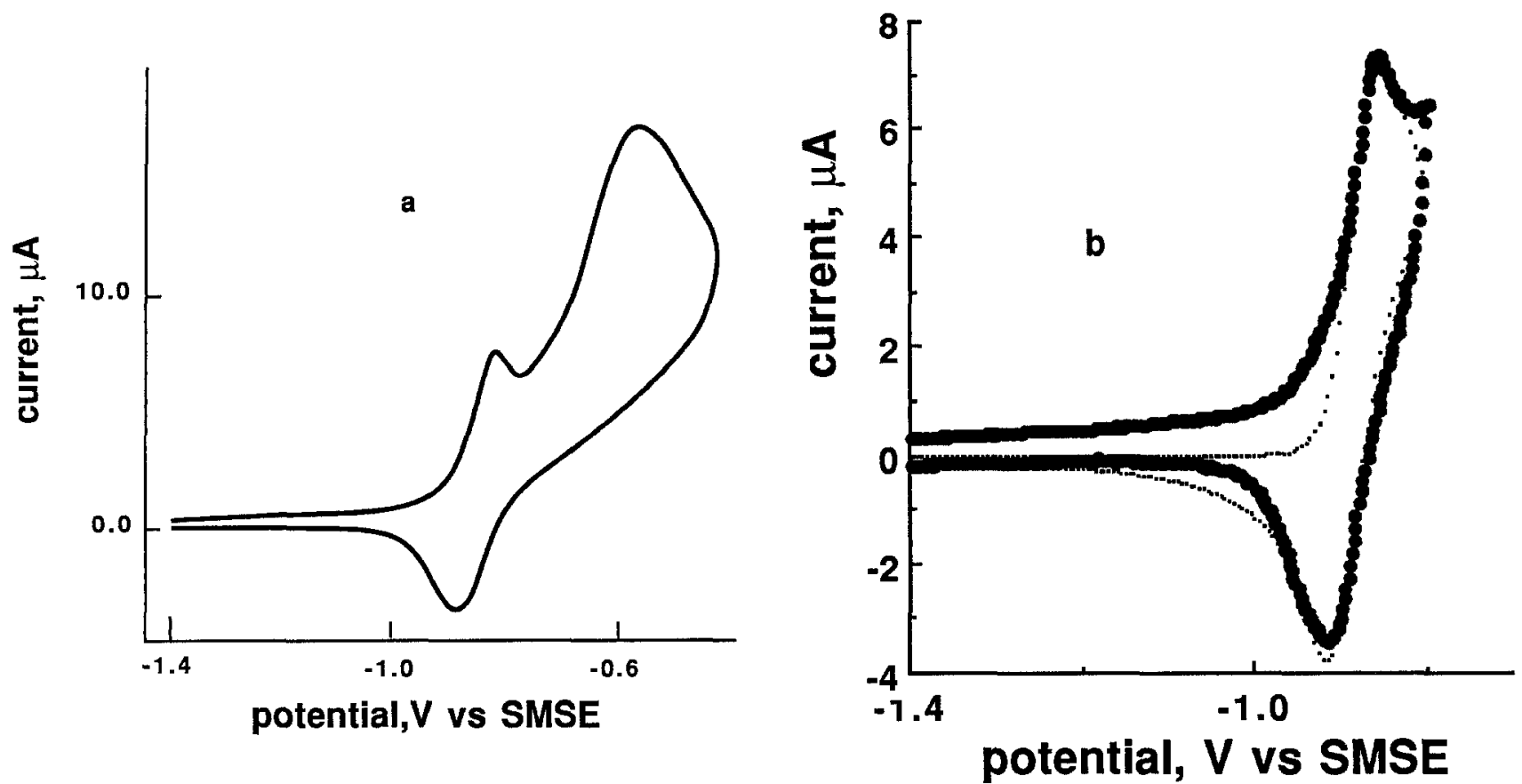

Fig. 4. (a) As in Fig. $1 ; v=200 \mathrm{~V} / \mathrm{s}$; (b) points represent the same curve, but with the reversal potential $E_{\lambda}=-0.8 \mathrm{~V}$. Darker curve: $\mathrm{CV}$ computed for an $E_{q} C_{i}$ mechanism (simulation parameters as in Fig. 3).

At $v$ of about $200 \mathrm{~V} / \mathrm{s}$, the ratio of their heights, $i_{\mathrm{pc}} / i_{\mathrm{pa}}$, tends to unity (Fig. 4a). When the reversal potential was placed between the two anodic peaks (Fig. 4b), the height and the position of the cathodic peak were the same as those with reversal after the second anodic peak. Therefore this cathodic peak is due to the reduction of some product of the first stage of the electro-oxidation. The position of this pair of peaks (which depends slightly on scan rate) and the $i_{\mathrm{pd}} d i_{\mathrm{pa}}$ ratio suggests quasi-reversibility of the first electron transfer. Applying the well-known equation of a reversible voltammogram ${ }^{20}$ to the anodic peak current, we have found $n \approx 1.8$, which is in good agreement with $n=2$, a value that has been proposed for the first stage of borohydride oxidation ${ }^{3,8}$. The product of this stage is very unstable and accounts for the absence of a cathodic peak in slow-scan voltammograms. This intermediate cannot be $\mathrm{BH}_{3} \mathrm{OH}^{-}$, which was proposed previously ${ }^{3,4}$ and also forms during hydrolysis, because that ion is fairly stable (the half-life time in an alkaline medium was estimated to be more than $3 \mathrm{~h}^{5}$ and is nonreducible in the given potential region? ${ }^{7}$ Our $\mathrm{CV}$ is different from the voltammogram of the mixture of $\mathrm{BH}_{4}^{-}$and $\mathrm{BH}_{3} \mathrm{OH}^{-}$shown in Ref. $7 \mathrm{c} v=$ $5.56 \mathrm{mV} / \mathrm{s}$ ), where strong adsorption of $\mathrm{BH}_{3} \mathrm{OH}^{-}$and two anodic peaks are observed. We believe the most likely mechanism of this stage is the ECE sequence

$$
\begin{gathered}
\mathrm{BH}_{4}^{-}-e^{-} \rightleftarrows \mathrm{BH}_{4}^{\cdot} \\
\mathrm{BH}_{4}^{\cdot}+\mathrm{OH}^{-} \rightleftarrows \mathrm{BH}_{3}^{-}+\mathrm{H}_{2} \mathrm{O} \text { (very fast) } \\
\mathrm{BH}_{3}^{-}-e^{-} \rightleftarrows \mathrm{BH}_{3}
\end{gathered}
$$

This mechanism, analogous to the sequence found for quinone/hydroquinone, is similar to that suggested by Tasaka et $a .^{8}$ for this process at a Pd electrode (Pd electrodeposited on a porous $\mathrm{Ni}$ substrate). However, they did not see any reversible stage, since their technique was not fast enough to detect the 2 e-reaction product. Monoborane should react rapidly with water or $\mathrm{OH}^{-21}$ and dimerize to diborane. The products of these reactions presumably are oxidized further to finally produce an $8 e^{-}$wave. The disappearance of $\mathrm{BH}_{3}$ results in the irreversibility seen at slow scan rates.

Scanning electrochemical microscopy.-When used in the G/C mode, a SECM can detect at the tip electrode products or intermediates generated at the substrate (or vise versa) in a manner analogous to the approach used at the rotating ring-disk electrode. The basic approach used is shown in Fig. 5. In this study we used SECM to find out whether the intermediates generated in the $2 e^{-}$wave react on the anode surface or diffuse into the solution and participate in a homogeneous reaction. If the intermediates leave the substrate, the $\mathrm{G} / \mathrm{C}$ experiment described above should show a reduction current at the tip electrode. A plot of the currents at the substrate $\left(i_{\mathrm{S}}\right)$ and tip $\left(i_{\mathrm{T}}\right)$ in the SECM $\mathrm{G} / \mathrm{C}$ mode as a function of the potential are given in Fig. 6. As expected, the cathodic $i_{\mathrm{T}}$ was observed only when the substrate potential was in the region of borohydride oxidation. Obviously, this effect can be observed only if the tip/ substrate distance is so small that the intermediate particle

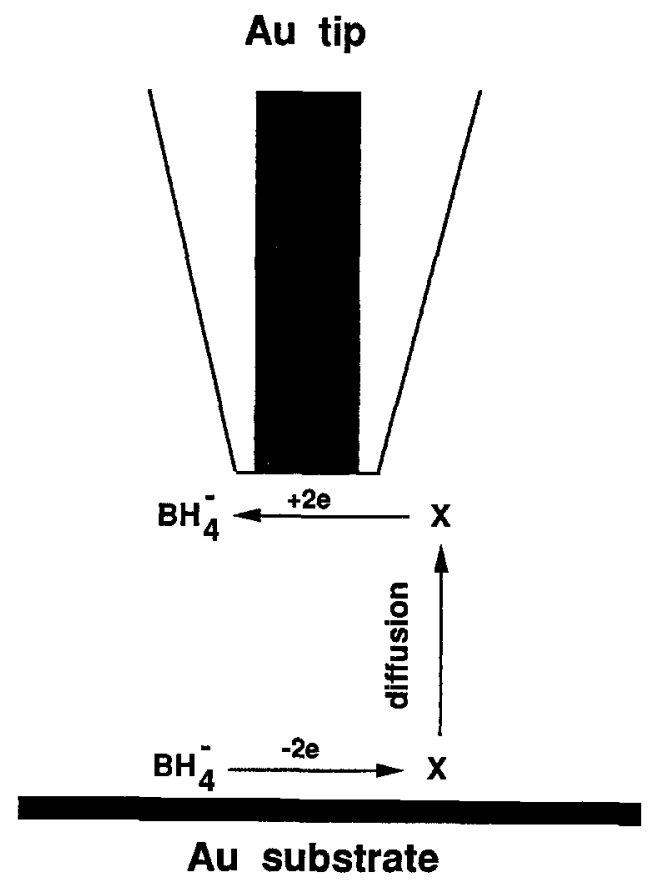

Fig. 5. Schematic diagram of the SECM experiment (not drawn to scale) in the generation/collection mode. The intermediate $X$ generated at the substrate electrode during the first stage of borohydride oxidation is then collected ot the tip electrode. 
substrate potential, $V$ vs SMSE
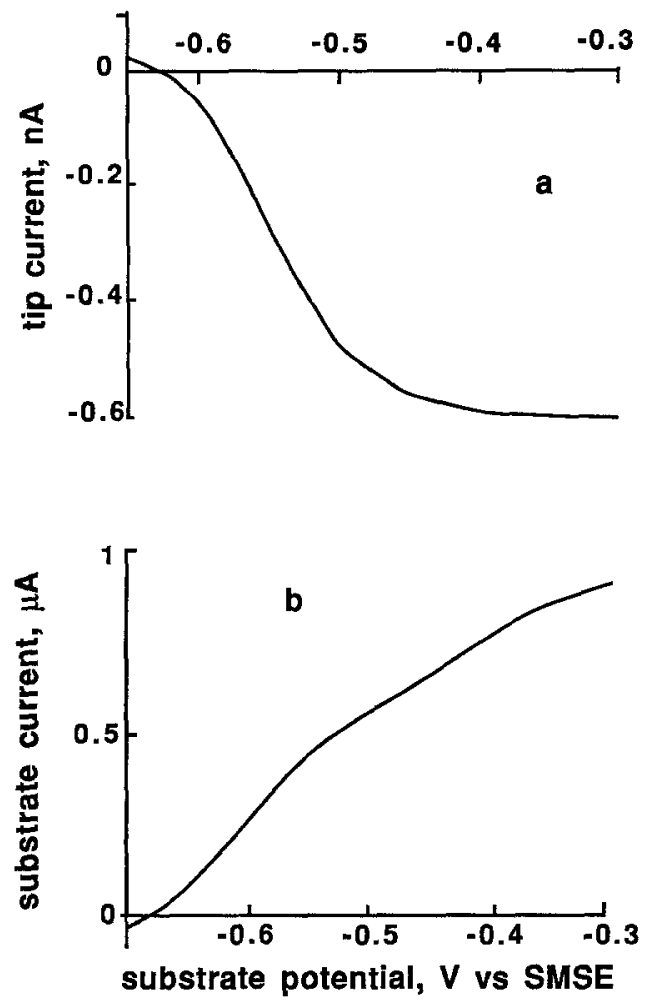

Fig. 6. Dependencies of the steady-state SECM tip (a) and substrate (b) currents vs. substrate potential in the $G / C$ mode. $10 \mu \mathrm{m}$ diam Au tip biased af $-1.3 \mathrm{~V}$ vs. SMSE and $100 \mu \mathrm{m}$ diam Au substrate are separated by $\sim 1 \mu \mathrm{m}$. The electrolyte is the same as in Fig. 1.

can traverse the gap during its life time. The life time $(\tau)$ can be estimated from the following inequality

$$
d \leq \sqrt{D \tau}
$$

Taking $d=1 \mu \mathrm{m}$ and the diffusion coefficient $D=10^{-5}$ $\mathrm{cm} / \mathrm{s}$, one obtains $\tau \geq 1 \mathrm{~ms}$, which corresponds to the first (or pseudo-first) order rate constant of the order of $500 \mathrm{~s}^{-1}$ or less.

Digital simulations of voltammograms.--To gain a better understanding of the reaction mechanism, and especially the stability and adsorption of the proposed interme- diate, digital simulations of the fast-scan CV were undertaken. The fast quasi-explicit finite-difference method (FQEFD) followed that of Feldberg ${ }^{22}$ and assumed that the sequence given in Eq. 5-7 could be considered as a single $2 e^{-}$transfer reaction, e.g., Eq. 9 (since quasi-reversible behavior is seen at fast scan rates), followed by irreversible decomposition of $\mathrm{BH}_{3}$

$$
\mathrm{BH}_{4}^{-}+\mathrm{OH}^{-} \rightleftarrows \mathrm{BH}_{3}+\mathrm{H}_{2} \mathrm{O}+2 e^{-}
$$

The decomposition of intermediate was assumed to produce no species that were electroactive at the potentials of the first $(n=2)$ wave. Thus the situation was approximated by an $E_{\mathrm{q}} C_{\mathrm{i}}$ mechanism for the first stage of the oxidation. The following values of the standard electrochemical rate constant, $k_{\mathrm{s}}=0.27 \mathrm{~cm} / \mathrm{s}$, the transfer coefficient, $\alpha=0.5$, the formal potential, $E^{\circ \prime}=-0.886 \mathrm{~V} v s$. SMSE, and the decomposition rate constant of the intermediate, $k=250 \mathrm{~s}^{-1}$ provided an acceptable fit of the theoretical and experimental curves for $v$ from $50 \mathrm{~V} / \mathrm{s}$ to $200 \mathrm{~V} / \mathrm{s}$ (Fig. $3 \mathrm{~b}, 4 \mathrm{~b}$ ). However, for scan rates at or above $500 \mathrm{~V} / \mathrm{s}$, significant deviations between experiment and theory occur. Coincidence between the experimentally obtained $i_{\mathrm{pa}} / v^{1 / 2} v s . v^{1 / 2}$ plot and the theoretical curve for $E_{\mathrm{a}} C_{\mathrm{i}}$ mechanism (where, the current function, $x=i_{\mathrm{p}} /\left(F A c^{*} \sqrt{F D v / R T}\right) ; c^{*}$ is the bulk concentration) is also observed at $v \leq 200 \mathrm{~V} / \mathrm{s}$ (Fig. 7a). At higher scan rates, $\chi$, rather than being only slightly dependent on $v$, increases linearly with $v^{1 / 2}$. This is a well-known indication of reactant adsorption ${ }^{23,24}$. This fact, together with the analogous linear dependence of $i_{\mathrm{pc}} / v^{1 / 2} v \mathrm{~s}$. $v^{1 / 2}$ (Fig. 7b) at high $v$, suggests that adsorption of both participants occurs during this electrochemical stage. However, there are no specific criteria for determining from the $\mathrm{CV}$ data whether $\mathrm{O}$, $R$, or both species are adsorbed, even in the case of uncomplicated electron transer ${ }^{24}$. The coverage of the electrode by the adsorbed species can be estimated by the equation ${ }^{25}$

$$
\Gamma^{*}=\frac{4 R T i_{\mathrm{pa}}}{n^{2} F^{2} v A}=\frac{4(R T D)^{1 / 2} c^{*} \chi_{\mathrm{a}}}{n^{2} F^{1 / 2} v^{1 / 2}}
$$

Substitution of the $\mathrm{X}_{\mathrm{a}} / v^{1 / 2}$ value, which is equal to the slope of the linear part of the curve $7 \mathrm{a}$, into Eq. 10, yields $\Gamma^{*}=$ $4 \times 10^{-10} \mathrm{~mol} / \mathrm{cm}^{2}$. Now one can estimate the fractional surface coverage using the formula

$$
\theta=\Gamma^{*} N_{\mathrm{A}} S
$$

where $N_{\mathrm{A}}=6.02 \times 10^{23} \mathrm{~mol}^{-1}$ is Avogadro's number and $S$ is the cross-sectional area of an adsorbed species. The last parameter can be assumed to be no more than $3 \times 10^{-19}$ $\mathrm{cm}^{2}$ using the values of B-H bond length $=1.25 \AA$ and
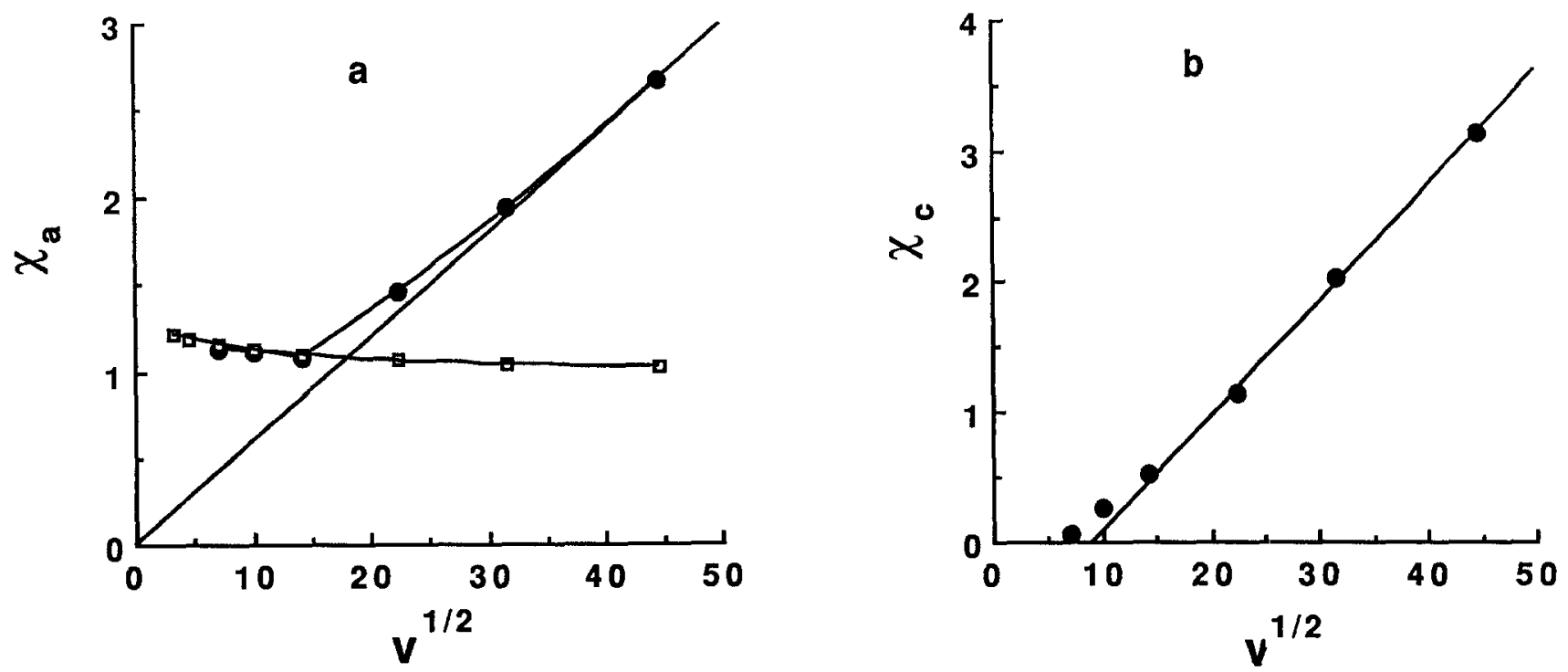

Fig. 7. Dependences of the anodic (a) and cathodic (b) peak currents upon the scan rate for the first stage of borohydride oxidation. Squares represent the simulated working curve for an $E_{\mathrm{q}} C_{i}$ mechanism; closed circles represent experimental data. The slope of the straight line (a) yields the value of $\Gamma^{*}$. The dimensionless current function is $\chi=i_{\mathrm{p}} /\left(F A c^{\circ} \sqrt{F D v / R T}\right)$. For simulation parameters, see Fig. 3. 


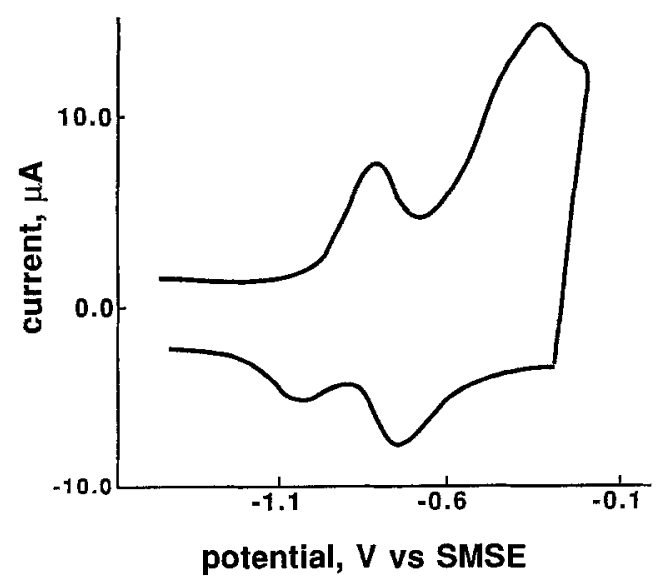

Fig. 8. CV at a $12.5 \mu \mathrm{m}$ diam Au microdisk in $10 \mathrm{mM}$ sodium borohydride, $1 \mathrm{M} \mathrm{NaOH} ; \mathrm{v}=10^{4} \mathrm{~V} / \mathrm{s}$.

the atomic radius $H \cong 0.3 \AA^{26}$, and assuming a close-packed arrangement of $\mathrm{BH}_{4}^{-}$(without associated cations on the surface). Substituting the calculated $S$ and $\Gamma^{*}$ values into Eq. 11, we find $\theta \cong 10^{-4}$. Although the fractional surface coverage is small, indeed almost negligible by usual electrochemical standards, it has a very large effect on the CV behavior at high scan rates. This extreme sensitivity to fractional adsorption can be an important limitation in very-high-scan-rate $\mathrm{CV}$ at ultramicroelectrodes. From the equations for the peak current of a nernstian CV wave and that of an adsorbed material we can write ${ }^{25}$

$$
\frac{i_{\mathrm{p}}(\mathrm{ads})}{i_{\mathrm{p}}(\mathrm{rev}, \mathrm{diff})}=\frac{n^{1 / 2} F^{2} v^{1 / 2} \Gamma^{*}}{4 R T D^{1 / 2} \mathrm{c}^{*} \times 2.69 \times 10^{-5}}
$$

Substituting the above values of the kinetic parameters into Eq. 12 and assuming $\theta=10^{-4}$, one can conclude that $i_{\mathrm{p}}$ (ads) $\geq 5 i_{\mathrm{p}}$ (rev,diff) at $v=10 \mathrm{kV} / \mathrm{s}$.

Further increases in scan rate (Fig. 8) led to the appearance of the second cathodic peak, indicating that a second stage of the oxidation is also reversible. Since the scan rate necessary for observing the reduction of this intermediate is about 100 times higher than that of the previous case, the chemical reaction responsible for kinetic irreversibility of this stage is much faster. Unfortunately, at a scan rate of about $10^{4} \mathrm{~V} / \mathrm{s}$ we are still unable to split the main anodic peak which should correspond to a six-electron transfer process. At the higher $v$, this peak displaces to a more extreme potential region where the oxidation of gold occurs. Therefore in the faster voltammogram one can see only the initial two-electron stage (Fig. 9). These reversible chargetransfer processes cannot be attributed to hydrogen evolution $^{3,6}$, because this reaction is irreversible on gold electrodes.

\section{Conclusions}

The electro-oxidation of borohydride in strongly alkaline media has traditionally been described as a thermodynamically irreversible process ${ }^{1}$. However, by fast CV one can distinguish at least two reversible electrochemical stages. The overall (kinetic) irreversibility of the process is caused by very unstable intermediate products that can be reduced only under conditions of a sufficiently fast electrochemical method. Although the process forming this intermediate is a $2 e^{-}$process, the first intermediate cannot be the rather stable $\mathrm{BH}_{3} \mathrm{OH}^{-}$, as previously suggested. Instead we propose a borane-species, e.g., $\mathrm{BH}_{3}$, or $\mathrm{B}_{2} \mathrm{H}_{6}$. SECM experiments showed that this intermediate is not totally adsorbed on the electrode, but rather diffuses into the solution and participates in a rapid homogeneous chemical reaction. The kinetic parameters of the first twoelectron stage of the electro-oxidation determined by means of digital simulation are $k_{\mathrm{s}}=0.3 \mathrm{~cm} / \mathrm{s}, \alpha=0.5, E^{\circ}=$ $-0.89 \mathrm{~V} v \mathrm{~s}$. SMSE; and the first (or pseudo-first) order rate constant for intermediate decomposition is $k=250 \mathrm{~s}^{-1}$. At scan rates faster than $200 \mathrm{~V} / \mathrm{s}$, the adsorption of the electro-

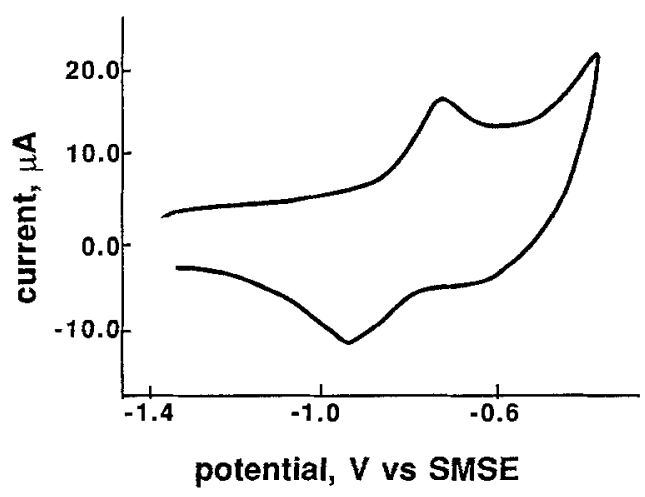

Fig. 9. As in Fig. $8 ; v=3 \times 10^{4} \mathrm{~V} / \mathrm{s}$. The second anodic peak has been displaced in a potential region of gold oxidation.

active species associated with the first two-electron stage of the oxidation becomes apparent and leads to the linear dependence of the peak current on scan rate. A very small surface coverage (estimated to be less than 0.001 ) is shown to be enough to produce adsorption- rather than diffusioncontrolled voltammetric behavior. The rate of a second chemical stage is much higher and could not be determined in these experiments.

\section{Acknowledgments}

The support of this research by SACHEM and NSF (CHE8901450) is gratefully acknowledged. Our thanks to Dr. D. O. Wipf, who built the SECM apparatus used in this work.

Manuscript submitted Jan. 15, 1992; revised manuscript received April 23, 1992.

The University of Texas at Austin assisted in meeting the publication costs of this article.

\section{LIST OF SYMBOLS}

A electrode surface area, $\mathrm{cm}^{2}$

a radius of a disk electrode, $\mathrm{cm}$

$c^{*} \quad$ bulk concentration, $\mathrm{mol} / \mathrm{cm}^{3}$

$D$ diffusion coefficient, $\mathrm{cm}^{2} / \mathrm{s}$

$E$ instantaneous value of the electrode potential, $\mathrm{V}$

$E^{\circ} \quad$ standard (formal) potential, V

$F \quad$ Faraday

$i_{\mathrm{T}} \quad$ microdisk steady-state current, $A$, equal to $4 n F c^{*} \mathrm{Da}$

$i_{\mathrm{pc}}, i_{\mathrm{pa}} \quad$ cathodic and anodic peak currents, A

$k$ pc $i_{\text {pa }}$ first-order homogeneous rate constant, $\mathrm{s}^{-1}$

$k_{s} \quad$ standard rate constant for heterogeneous

et reaction, $\mathrm{cm} / \mathrm{s}$

d distance between dip and substrate electrodes in SECM, cm

$n$ number of electrons involved in electrode reaction

$S \quad$ cross-sectional area of an adsorbed species, $\mathrm{cm}^{2}$

$v \quad$ scan rate, $\mathrm{V} / \mathrm{s}$

$\alpha \quad$ transfer coefficient

$\Gamma^{*} \quad$ coverage of the electrode by the adsorbed species, $\mathrm{mol} / \mathrm{cm}^{2}$

$\theta$ fractional surface coverage

$x \quad$ dimensionless current function

$\underset{7}{ }$ intermediate life time, $s$

\section{REFERENCES}

1. J. H. Morris, H. J. Gysling, and D. Reed, Chem Rev., 85 , 51 (1985).

2. E. D. Marshall and R. A. Widing, U.S. AEC Report, A.E.C.D., p. 2914 (1950).

3. J. P. Elder and A. Hickling, Trans. Faraday Soc., 58, 1852 (1962).

4. J. P. Elder, Electrochim. Acta, 7, 417 (1962).

5. J. A. Gardiner and J. W. Collat, Inorg. Chem., 4, 1208 (1965); J. Am. Chem. Soc., 87, 1692 (1965).

6. A. Kinjo, Diss. Abstr. B, 29, 3235 (1969).

7. Y. Okinaka, This Journal, 120, 739 (1973).

8. A. Tasaka, T. Tojo, M. Kubokawa, and K. Shimizu, Denki Kagaku Oyobi Kogyo Butsuri Kagaku,48, 123 
(1980); ibid., 48, 606 (1981). Chem. Abstr., 93, 15644g (1980); ibid., 94, 73452z (1981).

9. M. V. Mirkin and A. J. Bard, Anal. Chem., 63, 532 (1991).

10. G. Denuault, M. V. Mirkin, and A. J. Bard, J. Electroanal. Chem., 308, 27 (1991).

11. D. O. Wipf and A. J. Bard, This Journal, 138, 496 (1991)

12. H. Yang and A. J. Bard, J. Electroanal. Chem., 306, 87 (1991).

13. A. J. Bard, F.-R. F. Fan, J. Kwak, and O. Lev, Anal. Chem., 61, 132 (1989).

14. R. C. Engstrom, T. Meany, R. Tople, and R. M. Wightman, ibid., 59, 2005 (1987).

15. F. Zhou, P. R. Unwin, and A. J. Bard, J. Phys. Chem., In press.

16. J. Kwak and A. J. Bard, Anal. Chem., 61, 1794 (1989).

17. P. R. Unwin and A. J. Bard, J. Phys. Chem., 95, 7814 (1991).
18. A. J. Bard, M. V. Mirkin, P. R. Unwin, and D. O. Wipf, ibid., 96, 1861 (1992).

19. J. Kwak and A. J. Bard, Anal. Chem., 61, 1221 (1989)

20. R. S. Nicholson and I. Shain, ibid., 37, 706 (1964).

21. A. Stock, Hydrides of Boron and Silicon, p. 143, Cornell University Press, Ithaca, NY (1957).

22. S. W. Feldberg, J. Electroanal. Chem., 290, 41 (1990).

23. R. H. Wopschall and I. Shain, Anal. Chem., 39, 1514 (1967).

24. E. Laviron, in Electroanalytical Chemistry, Vol. 12, A. J. Bard, Editor, p. 53, Marcel Dekker, Inc., New York (1969).

25. A. J. Bard and L. R. Faulkner, Electrochemical Methods, p. 522, Wiley, New York (1980).

26. Comprehensive Inorganic Chemistry, J. C. Bailar, Jr., H. J. Emeleus, R. Nyholm, and A. F. Trotman-Dickenson, Editors, Vol. 1, Pergamon Press, Ltd., Oxford (1973).

\title{
Characterization and Catalytic Activity of Covalently Linked Bipyridyl Ruthenium OXO Dimers
}

\author{
Helen Hurrell Petach and C. Michael Elliott* \\ Department of Chemistry, Colorado State University, Fort Collins, Colorado 80523
}

\section{ABSTRACT}

Covalently linked bipyridyl ligands (L-L) with alkyl linkages varying from $\left(-\mathrm{CH}_{2}-\right)_{2}$ to $\left(-\mathrm{CH}_{2}-\right)_{12}$ were used to prepare ruthenium oxo dimers of the form $\left[(\text { bpy })\left(\mathrm{H}_{2} \mathrm{O}\right) \mathrm{RuO}(\mathrm{L}-\mathrm{L}) \mathrm{Ru}\left(\mathrm{H}_{2} \mathrm{O}\right)(\mathrm{bpy})\right]^{4+}$. The bridging alkyl linkage increases the stability of these oxo dimers by maintaining the relative proximity of the two ruthenium centers even when the oxo bridge is cleaved. These complexes have been characterized by electronic spectroscopy and electrochemistry. The electrochemistry in $\mathrm{CH}_{3} \mathrm{CN} / 0.1 \mathrm{M}$ TBAP exhibits a reversible one-electron oxidation followed by a second reversible two-electron oxidation which becomes catalytic in aqueous solution. The catalytic generation of dioxygen from water was observed upon bulk electrolysis of the oxo dimer at $+1.39 \mathrm{~V}$.

The reaction of $2 \mathrm{H}_{2} \mathrm{O} \rightarrow \mathrm{O}_{2}+4 \mathrm{H}^{+}+4 e^{-}$is essential for the electron flow in photosynthesis which leads to the conversion of light to chemical energy. Ruthenium oxo dimers have been shown to be catalytically active in this reaction $^{1,2}$, an essential step in the emulation of photosynthesis. Meyer and co-workers ${ }^{1}$ report an active catalyst, $\left[(\mathrm{bpy})_{2}\left(\mathrm{H}_{2} \mathrm{O}\right) \mathrm{Ru}-\mathrm{O}-\mathrm{Ru}\left(\mathrm{H}_{2} \mathrm{O}\right)(\mathrm{bpy})_{2}\right]^{4+}$, (I), which has finite stability and thus limited catalytic activity due to decomposition of the Ru-O-Ru linkage by cleavage or anation.

The oxo bridge seems to be essential for the catalytic activity of this complex since analogous ruthenium complexes of $6,6^{\prime}$-dimethyl-2,2'-bipyridine and 2,9-dimethyl1,10-phenanthroline which cannot form an oxo bridge due to their steric bulk and are not catalytically active with respect to water oxidation ${ }^{3}$.

We now report on the electrochemistry and catalytic activity of ruthenium oxo dimers which incorporate a saturated alkyl bridge between bipyridines bound to each of the two ruthenium atoms. These complexes were prepared in an effort to increase the molecule's stability by reducing the entropic barrier to oxo bridge formation. Additionally, if the oxo bridge were cleaved, the alkyl linkage would hold the ruthenium centers in close proximity for reformation of the oxo bridge. The more stable complex anticipated should have a longer catalytic lifetime.

\section{Materials and Methods}

Reagents.-2,2'-Bipyridine (bpy) was used as received from Matheson. 4,4'-Dimethyl-2,2'-bipyridine $\left(4,4^{\prime}\right.$-dmb) was recrystallized from ethyl acetate. $\mathrm{Ru}(\mathrm{bpy}) \mathrm{Cl}_{4}$ was prepared as described previously ${ }^{4}$. The 1,5-bis $\left(4^{\prime}\right.$-methyl-2,2'bipyridyl-4-yl)pentane, 1,6-bis(4'-methyl-2,2'-bipyridyl-4yl) hexane, and 1,12-bis(4'-methyl-2,2'-bipyridyl-4-yl) dodecane were prepared as described previously ${ }^{5}$ and will be denoted (L-5-L), (L-6-L), and (L-12-L), respectively. The 1,2-

* Electrochemical Society Active Member. bis(5'-methyl-2,2'-bipyridyl-5-yl)ethane, denoted (L-2-L), was prepared in a manner similar to the other bisbipyridinealkane ligands except that the carbanion of $5,5^{\prime}$ dimethylbipyridine was treated with one-half equivalent of $I_{2}$ to effect the linkage ${ }^{6}$.

Gel permeation chromatography was performed with Sephadex G-25 as packing material.

$\left[(\mathrm{bp} y)(\mathrm{Cl})_{2} \mathrm{Ru}(\mathrm{L}-\mathrm{L}) \mathrm{Ru}(\mathrm{Cl})_{2}(\mathrm{bpy})\right]$ (II). $-\mathrm{Ru}(\mathrm{bpy}) \mathrm{Cl}_{4}$ $(0.200 \mathrm{~g} ; 0.501 \mathrm{mmol})$ and $(\mathrm{L}-12-\mathrm{L})(0.127 \mathrm{~g} ; 0.250 \mathrm{mmol})$ were refluxed in $10 \mathrm{ml}$ of DMF with a 10 times molar excess of $\mathrm{LiCl}$ for $7 \mathrm{~h}$. After cooling, acetone was added to the reaction mixture, and the precipitate was collected. The product was washed with water and methylene chloride and vacuum dried. The analogous complexes for the (L-5-L), (L-6-L), (L-2-L) bridging ligands were prepared similarly. Yields were typically $50 \%$ of theoretical.

$\left[(b p y)\left(\mathrm{H}_{2} \mathrm{O}\right) \mathrm{Ru}(\mathrm{O})(\mathrm{L}-\mathrm{L}) \mathrm{Ru}\left(\mathrm{H}_{2} \mathrm{O}\right)(\mathrm{bpy})\right]^{4+}(\boldsymbol{I I I})$.-This was prepared by a modified procedure of Rotzinger et al. ${ }^{7}$. II $(0.075 \mathrm{~g} ; 0.065 \mathrm{mmol})$ and $\mathrm{Ag}_{2} \mathrm{SO}_{4}(0.040 \mathrm{~g} ; 0.130 \mathrm{mmol})$ were heated to reflux in $0.5 \mathrm{M} \mathrm{H}_{2} \mathrm{SO}_{4}$. The reaction mixture was cooled to $40^{\circ} \mathrm{C}$ and stirred for $3 \mathrm{~h}$, after which time the solution became red, indicating that the chloride ligands had been displaced. The solution was filtered to removed $\mathrm{AgCl}(\mathrm{s})$ and electrolyzed at $+1.1 \mathrm{~V}$ using a platinum gauze electrode for $3 \mathrm{~h}$ at $40^{\circ} \mathrm{C}$. The electrolysis was followed spectroscopically and continued until the reaction mixture became blue-green in color at which time approximately two equivalents of charge had been passed. A saturated aqueous solution of $\mathrm{NaClO}_{4}$ was used to precipitate the product. The initial product was redissolved in acetonitrile and precipitated with ether. The precipitate was collected and vacuum dried. Yields were typically $10 \%$ of theoretical.

Characterization.-Molecular-weight determinations were carried out on the ruthenium oxo dimers to ascertain 